\title{
AVALIAÇÃO DO DESEMPENHO DAS ESTAÇÕES DE TRATAMENTO POR LODO ATIVADO NO GRANDE RECIFE ASSOCIADA COM ANÁLISE DE IMAGENS DOS MICRO- ORGANISMOS PRESENTES NO LODO ATIVADO
}

\author{
L. C. ZOBY JR. ${ }^{1}$, L. M. P. NETO ${ }^{2}$, T. F. DALPANE ${ }^{3}$, T. M. N. PAIVA ${ }^{1}$, M. C. L. da $\operatorname{SILVA}^{1}$ e M. \\ A. MOTTA SOBRINHO ${ }^{1}$ \\ ${ }^{1}$ Universidade Federal de Pernambuco, Departamento de Engenharia Química \\ ${ }^{2}$ Uninassau, Engenharia Ambiental \\ ${ }^{3}$ Universidade de Pernambuco, Engenharia Civil \\ E-mail para contato: lczoby@yahoo.com.br
}

\begin{abstract}
RESUMO - O processo de tratamento de efluentes por lodos ativados é um dos processos de tratamento mais difundido em todo o mundo, devido principalmente a qualidade do efluente obtido. Entretanto, trata-se de um processo biológico dependente da atividade bacteriana para a estabilização da matéria orgânica proveniente dos esgotos. O tratamento de esgotos é importante para que os efluentes retornem aos rios e fontes sem afetarem o meio ambiente. As estações de tratamento de esgoto (ETE) estudadas foram Caçote e Janga, sendo a primeira de pequeno porte e a outra de grande porte, ambas tratam efluentes residências e são localizadas no Grande Recife. Foi feita a caracterização do efluente na entrada e saída do tratamento para saber a eficiência das mesmas associando aos microrganismos presentes. Através da análise de imagem que hoje em dia representa uma ferramenta de rápida utilização com grande perspectiva para o gerenciamento e operação das ETE's, fazendo uma verificação das características qualitativas e quantitativas da biota microbiana das ETE's.
\end{abstract}

\section{INTRODUÇÃO}

O sistema de tratamento por lodos ativados foi posto em funcionamento pela primeira vez no começo do século XX, em Manchester na Inglaterra, para o tratamento de esgotos de maneira empírica. Os fundamentos teóricos desse processo só foram desenvolvidos posteriormente (Da Silva, 2008). Atualmente, esse processo é um dos mais utilizados mundialmente para o tratamento de efluentes domésticos e industriais caracterizados por carga orgânica biodegradável e substratos nitrogenados, e essa popularidade se deve por possuir eficiência entre 95 e $98 \%$ de redução da poluição (Hammer e Hammer, 2006; Wiesmann et al., 2007). O princípio deste processo está ligada à oxidação bioquímica de diversos poluentes orgânicos e inorgânicos a partir da participação efetiva de uma variada biomassa microbiana, suspensa no meio e mantida sob concentração constante de oxigênio dissolvido (Bento et al, 2005; Mesquita et al., 2013).

Um exame microscópio do lodo ativado revela que ele é formado por uma população 


\section{9 a 22 de outubro de 2014 \\ Florianópolis/SC}

heterogênea de micro-organismos, responsáveis pela biodegradação dos substratos orgânicos. De fato, o lodo ativado ou microfauna dispersa é um complexo sistema composto por bactérias, protozoários, fungos e micrometazoários que se agregam sob a forma de flocos biologicamente ativos que fixam substratos complexos ou simples (Davies, 2005; Zoby Jr., 2011). A comunidade estabelecida é dinâmica e fundamental ao tratamento, sendo que cada espécie tem sua importância para o bom funcionamento do sistema (Amman et al, 1997). Logicamente, a boa eficiência de certo sistema de lodos ativados depende primordialmente da capacidade de floculação da biomassa ativa e da sua composição, além de outros fatores envolvidos no processo de oxidação (Bento et al, 2005).

A população microbiana pode sofrer as variações do sistema, e mudar continuamente de acordo com a variabilidade na composição química do esgoto e condições ambientais, constituindo-se num importante parâmetro do estado de funcionamento do processo, sendo assim um bom indicador da qualidade biológica do sistema. As bactérias são os micro-organismos mais importantes no sistema de tratamento por lodos ativados. Os fungos, os protozoários e os metazoários têm um papel secundário no processo de oxidação. O bom equilíbrio entre estes dois grupos produz flocos sãos, com boa propriedade de decantação e compressão, além de se obter um efluente final com baixa turbidez e baixa concentração de material em suspensão (Da Motta et al., 2001). Altas concentrações de protozoários no tanque de aeração normalmente indica um bom desempenho da estação de tratamento. A distribuição e abundância das espécies de fauna têm sido apontados como indicadores da qualidade do tratamento dos efluentes, fornecendo um mecanismo útil e rápido para avaliar o seu desempenho do tratamento (Ginoris et al., 2007).

O estudo das comunidades microbianas, através da identificação de protozoários por análise microscópica já vem sendo utilizado em estações de tratamento na Europa e nos Estados Unidos, assim como a quantificação das bactérias filamentosas responsáveis pelo intumescimento do lodo. Estes procedimentos são capazes de fornecer uma quantidade cada vez maior de dados para o controle das estações de tratamento de efluentes (Mesquita et al., 2013).

Papadimitriou et al. (2010) investigaram a correlação ente espécies de protozoários e parâmetros físico-químicos de monitoramento, como o pH, NO3, NH4, OD. Eles observaram que a diversidade de protozoários aumentava com o aumento da carga orgânica e do nitrogênio inorgânico e que a presença deles é fundamental para uma boa qualidade do efluente final. Já Esteban et al. (1991), ao avaliar a dinâmica da microfauna em procesos de lodo ativado, empregaram a análise de componentes principais e obtiveram conclusões interessantes sobre a microfauna, como que a $V$. micróstoma ao filtrar as bactérias, reduz a carga orgânica e que a O. microdiscum possui uma forte correção com o SBI (Slude Biotic Index).

Este trabalho trata-se de um estudo preliminar de eficiência de biodegradação de ETE por lodos ativados do Grande Recife, sendo avaliados parâmetros físico-químicos em amostras de efluentes domésticos e de lodo ativado concomitantemente a identificação da microfauna presente por análise de imagem. Ele busca validar em uma região de clima tropical com hábitos distintos da europa, os resultados obtidos por Madoni et al. (1993), que serviram de base para o desenvolvimento do índice biótico de lodos (SBI) (MADONI, 1994), o qual permite avaliar a eficiência de uma ETE a partir do estudo de sua microfauna. 


\section{MATERIAIS E MÉTODOS}

\subsection{Sítios de estudo, coleta e caracterização das amostras}

As amostras de efluente doméstico foram coletadas em duas estações de tratamento de lodos ativados, a ETE Caçote de pequeno porte, localizada na zona sul do Recife (latitude: $-8^{\circ} 5^{\prime} 56.72$ ”; longitude: -34 55'32.61'), e a ETE-Janga de grande porte, localizada no bairro de mesmo nome no município de Paulista (latitude: -792'76.97’, longitude: -3484'70.88”). As amostras dos efluentes foram coletadas na entrada e saída da estação para verificar a eficiência do tratamento através da caracterização; e uma amostra de lodo ativado na saída do tanque de aeração para estudo da microfauna presente. Todas as amostras coletadas foram acondicionadas em recipientes limpos e adequados (frasco de polietileno). O transporte das amostras até o laboratório foi realizado em ambiente refrigerado (isopor com gelo) com a finalidade de retardar a decomposição do conteúdo orgânico pelos microrganismos.

Nas amostras de efluente foram realizadas análises para caracterização da poluição presente. A determinação da DQO (Demanda Química de Oxigênio) foi realizada através do método colorimétrico e para DBO (Demanda Biológica de Oxigênio) foi utilizado o método manométrico. As demais análises para caracterização dos parâmetros globais, apresentadas na Tabela 1, foram executadas conforme os procedimentos analíticos do Standard Methods for the Examination of Water and Wastewater (APHA, 1995). Na amostra de lodo ativado coletada foram analisados o Índice Volumétrico de Lodo (IVL) e a série de sólidos voláteis para avaliação da concentração de biomassa presente nos flocos

Tabela 1 - Métodos de análise utilizados

\begin{tabular}{|l|l|l|}
\hline \multicolumn{1}{|c|}{ Parâmetros } & \multicolumn{1}{c|}{ Técnicas } & \multicolumn{1}{c|}{ Métodos } \\
\hline Turbidez & 2130 Turbidez & 2130B Método nefelométrico \\
\hline Alcalinidade & 2320 Alcalinidade & 2320B Método da titulação \\
\hline Condutividade & 2510 Condutividade & 2510B Método laboratorial \\
\hline SST & 2540 Sólidos & 2540B Sólido suspenso total seco a 103-105 ${ }^{\circ} \mathrm{C}$ \\
\hline SSF e SSV & $\begin{array}{l}\text { 4500-H+ Valor de } \\
\mathrm{pH}\end{array}$ & 2540E Sólidos suspensos fixos e voláteis $\left(550^{\circ} \mathrm{C}\right)$ \\
\hline $\mathrm{pH}$ & $4500-\mathrm{NTK}$ & 4500B Método Macro-Kjeldahl \\
\hline $\begin{array}{l}\text { Nitrogênio Total de } \\
\text { Kjeldahl }\end{array}$ & $\begin{array}{l}\text { 4500-NH3 } \\
\text { Nitrogênio (amônia) }\end{array}$ & 4500-NH3 Método titulométrico \\
\hline $\begin{array}{l}\text { Nitrogênio } \\
\text { amoniacal }\end{array}$ & Método Merck & Método Merck \\
\hline Nitrato/Nitrito & \multicolumn{2}{|l}{} \\
\hline
\end{tabular}

\subsection{Sistema de aquisição de imagens}

O sistema de aquisição de imagem (Figura 1) é formado por um microscópio óptico Axiostar 
Plus (Zeiss, Göttingen, Germany), sobre o qual é conectada uma câmara modelo SDC-312 (Samsung, Korea). A imagem é digitalizada para o computador através de uma placa de aquisição Pinnacle Sudio Movie Box HD.

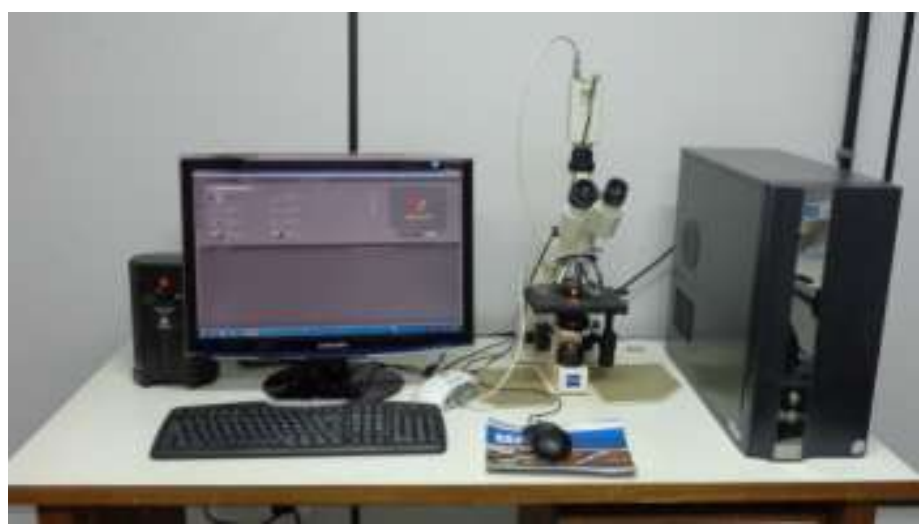

Figura 1 - Sistema de aquisição de imagens.

Uma gota da amostra foi depositada sobre uma lâmina, em estado fresco e visualizada em um microscópio óptico em campo claro com uma ampliação de 100 vezes. Para a aquisição das imagens dos flocos foi usada uma câmera de vídeo fixada sobre o microscópio e conectada a um computador por carta de aquisição.

\section{RESULTADOS}

\subsection{Caracterização das amostras de efluente na entrada e saída da ETE}

Na Tabela 2 estão apresentados os resultados obtidos na caracterização dos parâmetros físicoquímicos para os efluentes analisados, na entrada da caixa de areia e saída do decantador secundário das estações estudadas.

Esta tabela apresenta os resultados da determinação de matéria orgânica, tais quais DQO, $\mathrm{DBO}_{5}$, bem como os resultados das determinações da série dos sólidos, seguidos pela determinação da série de nitrogênio, condutividade, cor, $\mathrm{pH}, \mathrm{OD}$, turbidez, alcalinidade e o índice volumétrico de lodo. Convém lembrar que as amostras de efluente tratado foram coletadas no mesmo momento das amostras de efluente bruto, e nesse caso como não foi considerado o tempo de retenção hidráulico do processo, não sendo possível uma comparação direta dos valores dos parâmetros da entrada e saída das ETE.

Mesmo sendo uma estação de grande porte as amostras analisadas na entrada da ETE-Janga, não foram verificadas contribuições expressivas em termos de DQO, ao contrário do que apresentou a ETE-Caçote. Esta mesmo sendo de pequeno porte recebendo uma carga orgânica de aproximadamente 5 vezes maior que a ETE-Janga.

As amostras da ETE-Janga apresentaram baixos valores de ST (menores que $700 \mathrm{mg} . \mathrm{L}^{-1}$ ) o que 
está em concordância com os baixos valores observados para a DQO medidos para essa estação. Já para a série de sólidos em suspensão (SST, SSV e SSF) foi verificado que as amostras apresentam uma alta concentração de poluição particulada. Já para a ETE-Caçote por apresentar valor alto de DQO à série dos sólidos apresentaram alta concentração de poluição particulada.

Tabela 2 - Resultado da caracterização dos efluentes e do lodo ativado.

\begin{tabular}{|c|c|c|c|c|c|c|}
\hline & \multicolumn{3}{|c|}{ ETE CAÇOTE } & \multicolumn{3}{|c|}{ ETE JANGA } \\
\hline Análises & Entrada & Saída & Lodo & Entrada & Saída & Lodo \\
\hline $\mathrm{DQO}\left(\mathrm{mg} \mathrm{O}_{2} \cdot \mathrm{L}^{-1}\right)$ & 1006,42 & 339,98 & & 194,96 & 95,6 & \\
\hline $\mathrm{DBO}\left(\mathrm{mg} \mathrm{O}_{2} \cdot \mathrm{L}^{-1}\right)$ & 600 & 70 & & 117,5 & 37,5 & \\
\hline Sólidos totais $\left(\mathrm{mg} \cdot \mathrm{L}^{-1}\right)$ & 888 & 659 & & 462,5 & 563 & \\
\hline Sólidos fixos $\left(\mathrm{mg} . \mathrm{L}^{-1}\right)$ & 504 & 422 & & 322 & 417 & \\
\hline Sólidos voláteis (mg.L $\mathrm{L}^{-1}$ ) & 384 & 237 & & 140,5 & 146 & \\
\hline Sólidos suspensos totais $\left(\mathrm{mg} . \mathrm{L}^{-1}\right)$ & 530 & 472 & 530 & 140 & 218 & 194 \\
\hline Sólidos suspensos fixos $\left(\mathrm{mg} \cdot \mathrm{L}^{-1}\right)$ & 34 & 94 & 92 & 108 & 198 & 146 \\
\hline Sólidos suspensos voláteis $\left(\mathrm{mg} . \mathrm{L}^{-1}\right)$ & 496 & 378 & 438 & 32 & 20 & 48 \\
\hline Nitrogênio total Kjeldahl (mg. $\left.\mathrm{L}^{-1}\right)$ & 303,6 & 293,5 & & 89,26 & 96,7 & \\
\hline Nitrogênio amoniacal $\left(\mathrm{mg} . \mathrm{L}^{-1}\right)$ & 0,65 & 2,42 & & 0,83 & 3,19 & \\
\hline Nitrato $\left(\mathrm{mg} . \mathrm{L}^{-1}\right)$ & 20 & $0,9(<1,0)$ & & 0,9 & 0,8 & \\
\hline Condutividade $\left(\mu \mathrm{S} . \mathrm{cm}^{-1}\right)$ & 884 & 794 & & 561 & 706 & \\
\hline Cor $\left(\right.$ Pt.L $\left.^{-1}\right)$ & 340 & 249 & & 241 & 95 & \\
\hline pH (potencial hidrogeniônico) & 7,6 & 7,44 & & 6,48 & 6,6 & \\
\hline OD (oxigênio dissolvido) & $11 / 12$ & $07 / 08$ & & 17,1 & 16,97 & \\
\hline Turbidez & 345 & 203 & & 102 & 38,4 & \\
\hline Alcalinidade $\left(\mathrm{mg} \mathrm{Ca} \cdot \mathrm{L}^{-1}\right)$ & 298,34 & 282,36 & & 197,12 & 250,39 & \\
\hline $\begin{array}{l}\text { IVL (índice volumétrico de lodo) } \\
\left(\mathrm{mL} \cdot \mathrm{g}^{-1}\right)\end{array}$ & & & 18,868 & & & 79,38 \\
\hline
\end{tabular}

Os valores para as análises de NTK e nitrogênio amoniacal se apresentaram dentro dos valores médios observados para esgoto doméstico, para ambas as estações.

$\mathrm{O} \mathrm{pH}$ verificado para todas as amostra se manteve dentro da faixa de neutralidade a levemente alcalino (faixa de variação de 6,48 a 7,6). As análises de alcalinidade apresentaram valores acima da faixa típica para efluente doméstico (entre 197,12 e 298,34 $\mathrm{mg} \mathrm{CaCO}_{3} \cdot \mathrm{L}^{-1}$ ).

O índice volumétrico de lodo para a ETE Janga foi maior que para a ETE Caçote, ele representa 
o volume em mL ocupado por um grama de sólido em suspensão, indicando qualitativamente os padrões de sedimentabilidade do lodo. Caso o lodo esteja intumescido (excesso de filamentosas),

\subsection{Micro-organismos presentes no lodo ativado das estações}

Os microrganismos identificados presente no lodo ativado das estações estão apresentados na Tabela 4. Para os micro-organismos encontrados na ETE-Caçote: Opercularia sp. e Trachelophyllum indicam que o tratamento do efluente apresenta uma baixa qualidade do efluente; Trochilia sp., Epistylis sp., Trithigmostoma sp. estes indicam alta qualidade do efluente tratado; Nematode, Operculária sp. estes indicam pouca aeração no sistema; Epystilis sp. e Trochilia sp. a presença desses microrganismos apresenta uma boa nitrificação e a presença de Digononta indica que a idade do lodo é de 20 dias ou mais. Para a ETE-Janga: Epistylis sp., Trochilia sp., Monogononta e Arcella $s p$. Estes micro-organismos indicam alta qualidade do efluente tratado; Arcella sp., Monogononta e Trochilia sp.estes são indicadores que o sistema está com uma boa aeração e nitrificação; a presença de Arcella sp. e Monogononta indicam que a idade do lodo é de 20 dias ou mais. Essas classificações foram de acordo com Ginoris et al. (2007).

Como a análise é feita pelo grupo dominante, pode-se estão ponderar que o efluente da ETE Caçote (Figura 2) deve apresentar baixa qualidade, pois mesmo com uma elevada remoção de DQO o efluente final ainda possui uma DQO elevada. Já para a ETE Janga (Figura 3), a presença da Arcella e Monogononta estão de acordo, pois trata-se de um sistema da aeração prolongada, já a $V$. convalaria, um ciliado séssil, indica um efluente final de boa qualidade.

Tabela 3 - Micro-organismos presentes nas estações

\begin{tabular}{|l|l|}
\hline ETE-Caçote & ETE-Janga \\
\hline Aspidisca cicada & Aspidisca cicada \\
\hline Epystilis $s p$. & Epystilis sp. \\
\hline Closterium $s p$. & Closterium sp. \\
\hline Vorticella convalaria & Vorticella convalaria \\
\hline Ameba & Ameba \\
\hline Trochilia $s p$. & Trochilia sp. \\
\hline Digononta & Monogononta \\
\hline Nematode & Arcella sp. \\
\hline Opercularia $s p$. & \\
\hline Euplotes $s p$. & \\
\hline Trithigmostoma $s p$. & \\
\hline Paramecium & \\
\hline Trachelophyllum & \\
\hline Zoothamnium & \\
\hline
\end{tabular}




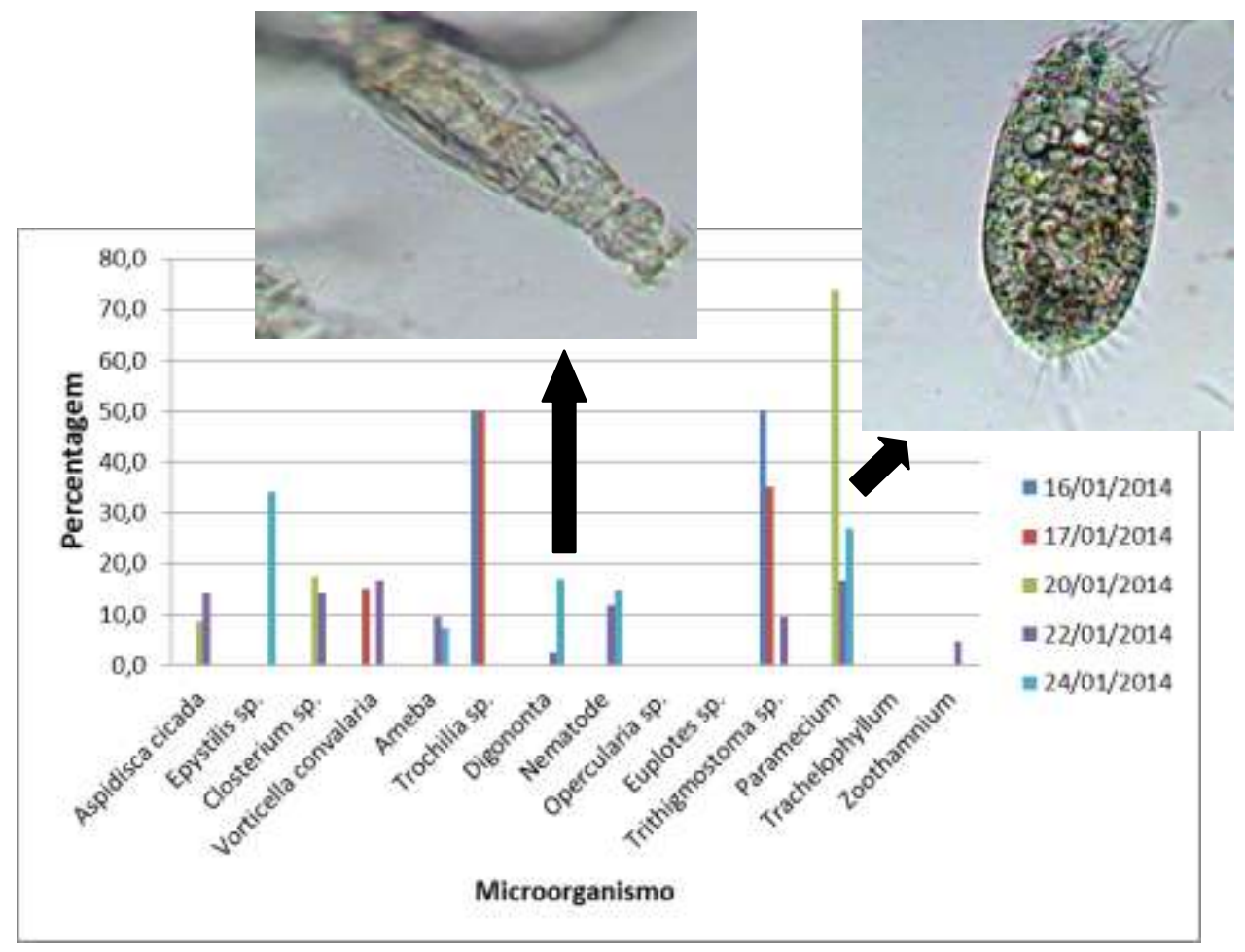

Figura 2 - Frequência de ocorrência dos microrganismos na ETE Caçote e foto de alguns deles.

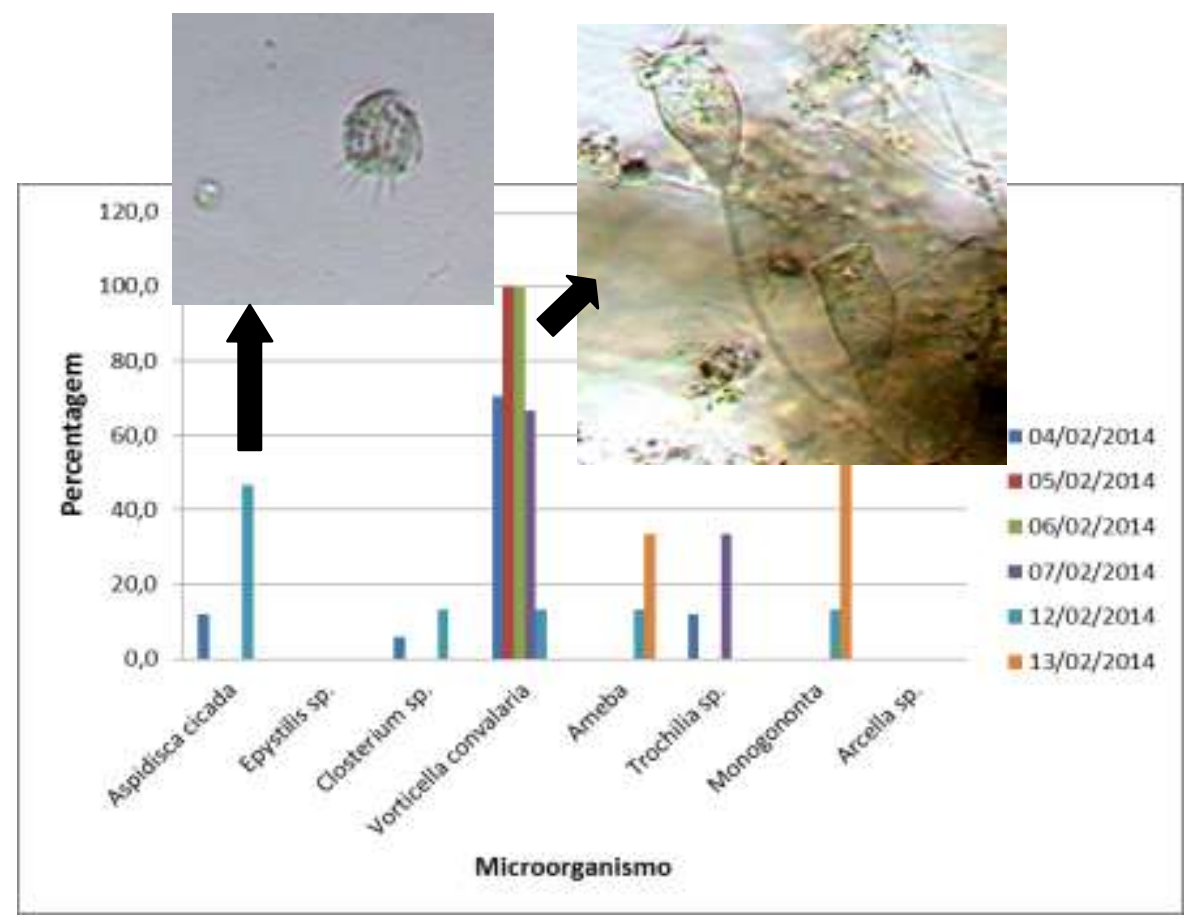

Figura 3 - Frequência de ocorrência dos microrganismos na ETE Caçote e foto de alguns deles. 


\section{9 a 22 de outubro de 2014 \\ Florianópolis/SC}

\section{CONCLUSÕES}

Os microrganismos presentes na ETE Janga apresentaram melhores contribuições para o desempenho da estação, apresentando uma remoção de DQO de 50,96\% comparada com a ETE Caçote que apresentou microrganismos que não seriam bons para o tratamento do efluente, ela apresentou uma remoção de 66,22\% em termos de DQO, mas uma DQO final elevada. Constatou-se assim que as correlações obtidas para ETEs na Europa foram válidas para as ETEs em estudo.

\section{REFERÊNCIAS}

AMMAN, R.; GLÖCKNER, F.O.; NEEF, A. Modern methods in subsurface microbiology: in situ identification of microorganisms with nucleic acid probes. FEMS Microb. Rev., v. 20, n. 3-4, p. 191-200, 1997.

APHA. Standard methods. 19th Edition. American Public Health Association, Washington, DC, 1995.

BENTO, A.P.; SEZERINO, P.H.; PHILIPPI, L.S.; REGINATTO, V.; LAPOLLI, F.R. Caracterização da microfauna em estação de tratamento de esgotos do tipo lodos ativados: um instrumento de avaliação e controle do processo. Eng. San. Ambient., v. 10, n. 4, p. 329-338, 2005.

DA MOTTA, M., PONS, M.N., ROCHE, N. Estudo da Decantabilidade dos Flocos de Lamas Ativadas de uma Estação de Tratamento de Efluentes por Análise de Imagem. Energ. Renov. y Med. Amb.,v.9,p. 43-49, 2001.

DA SILVA, Maria C.L.; Effet de la variabilité du fractionnement de la pollution carbonée sur le comportement des systèmes de traitement des eaux usées par boues activées. Tese de doutorado do Insitut National Politechnique de Lorraine. p. 206, 2008.

DAVIES, P.S. The biological basis of wastewater treatment. Glasgow, UK: Strathkelvin Instrument Ltd. 2005.

GINORIS, Y. P.; AMARAL, A. L.; NICOLAU, A.; COELHO, M. A. Z.; FERREIRA, E. C. Development of an image analysis procedure for identifying protozoa and metazoa typical of activated sludge system. Water Research, v. 41, p. 2581-2589, 2007.

HAMMER M.J., HAMMER Jr. , M.J. Water and Wastewater Technology, 5th edition, Prentice Hall of India, New Delhi, p. 540, 2006.

MADONI P. Protozoa in waste treatment systems. In Perspectives in Microbial Ecology (Edited by Megusar F. and Gantar M.), p. 86-90. Slovene Society of Microbiologists, Ljubljana. 1986.

MADONI, P. A sludge biotic index (SBI) for the evaluation of the biological performance of activated sludge plants based on the microfauna analysis. Wat. Res. v. 28, n. 1, p. 67-75, 1994.

MESQUITA, D. P.; AMARAL, A. L.; FERREIRA, E. C.; Activated sludge characterization through microscopy: Arevie won quantitative image analysis and chemometric techniques. Analytica Chimica Acta, v. 802, p. 14-28, 2013.

PAPADIMITRIOU, C.A., PAPATHEODOULOU, A., TAKAVAKOGLOU, V., ZDRAGAS, A., SAMARAS, P., SAKELLAROPOULOS, G.P., LAZARIDOU, M., ZALIDIS, G. Investigation of protozoa as indicators of wastewater treatment efficiency in constructed wetlands. Desalination, v. 250, n., p. 378-382, 2010.

WIESMANN, U.; CHOI, I. S.; DOMBROWSKI, E. M. Fundamentals of biological wastewater treatment.Weinheim: Willey-VCH Verlag GmbH \& Co., 355p,2007.

ZOBY JR., L. C. Aplicação do Modelo ASM1 na Simulação das Condições Operacionais de uma Estação de Tratamento de Efluentes por Lodo Ativado. Dissertação de mestrado da UFPE, 2011. 